\title{
Recollected experiences of first hospitalisation for acute psychosis among persons diagnosed with schizophrenia in South Africa
}

\author{
Y Zubi, BA (Hons), MA (Clin Psych); P Connolly, BA (Hons), MA (Couns Psych) \\ Department of Psychology, University of the Witwatersand, Johannesburg, South Africa
}

Corresponding author: Y Zubi (yumna.zubi@gmail.com)

\begin{abstract}
First-time admission to a psychiatric hospital for acute psychosis has been reported to be extremely traumatic, and this has not been adequately researched in the South African context. This study approached persons diagnosed with schizophrenia and explores their recollected, subjective experiences of their first admission to a South African psychiatric hospital ward for acute psychosis.

Semi-structured individual interviews were conducted with seven participants diagnosed with schizophrenia. These were analysed using thematic content analysis. While some participants had positive experiences, the majority reported frightening, distressing, emotionally painful or traumatic experiences during their first hospitalisation. The first hospital admission was also described as having serious longterm implications for the person's wellbeing, particularly as the diagnosis of schizophrenia made them feel isolated and stigmatised, which further hinders them from recovering from their symptoms and reintegrating into society.

This study therefore draws attention to aspects of the process of first admission to psychiatric wards in South Africa, which may benefit from proactive intervention and closer research attention.
\end{abstract}

S Afr J Psych 2013;19(3):81-85. DOI:10.7196/SAMJ.399

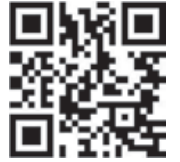
The psychiatric hospital is an important environment for the treatment of people who are severely psychotic. ${ }^{[1]}$ Hospitalisation is vital for a number of reasons: firstly, for providing care for acutely disturbed individuals, in an environment where medication can be quickly altered and side-effects closely monitored. Secondly, hospitalisation provides respite for patients from various stressors, ${ }^{[2]}$ as well as an escape from the society in which their behaviour produces friction. Furthermore, psychiatric hospitals fulfil many needs which family members cannot, such as ensuring treatment adherence. Many people diagnosed with schizophrenia often do not adhere to medication regimens, which puts them at risk of relapse. Thus, psychiatric hospitals help protect patients from themselves and others, while also helping them adhere to their medication. ${ }^{[3]}$

First-time admission to a psychiatric hospital has been reported to be very traumatic. ${ }^{[4]}$ Furthermore, some of the problems or trauma experienced during the first admission may be specific to a South African context. As Lund and Flisher explain, 'In the aftermath of apartheid, South Africa has inherited a fragmented, under-resourced and inequitable public-sector mental health service. ${ }^{[5]}$ South African psychiatric hospitals in general lack the resources to cater for the needs of people with psychiatric conditions. This is reflected in a range of areas, from the number of psychiatric beds available, to medication and the staff needed to maintain the operational needs of the psychiatric facility. ${ }^{[4]}$ Since this study focuses on a South African context, it will be useful to explore the particular challenges that South African hospitals face as suggested by the experiences of a first psychiatric hospital admission for acute psychosis of people diagnosed with schizophrenia.

\section{Contemporary challenges in the South African public health sector}

The South African public health sector serves $75-80 \%$ of the population. ${ }^{[6]}$ It is mainly sustained by very limited government funding. The distribution of highly skilled healthcare personnel is uneven, with a greater concentration in the private sector and urban areas than in the public sector and rural areas. The introduction of community service for healthcare professionals is intended to help alleviate this. ${ }^{[5]}$

South African hospital staff also face understaffing, staff attrition, and reduced job satisfaction. The shortage of mental health practitioners increases the workload of existing staff members, raising levels of stress, fatigue and emotional exhaustion, all of which compromise both the quality and safety of care. Staff members may have to work unpaid overtime to complete their workload, and may have less flexibility to take leave or participate in staff development and training activities. ${ }^{[7]}$ The day-to-day work in a psychiatric setting can be pressurised, which could lead to self-protective defences such as reductive labelling, us-and-them blame-throwing, and emotional detachment. ${ }^{[8]}$

\section{Psychiatric hospitals}

Regardless of the specific challenges within a South African context, first admissions to hospital for acute psychosis may be universally experienced as traumatic and damaging. There are a number of 
reasons for this. While psychiatric hospitalisation may be voluntary, it often is not, and in many cases people with mental illnesses may be forced into treatment by the police, judges or family members. ${ }^{[9]}$ To this day, debate continues over whether an infringement and deprivation of liberty can really be justified in order to help a patient into a rational state of being, or whether it is merely a means of social control over those who display deviant behaviour. ${ }^{[10]}$ Critics of involuntary commitment argue that it does more harm than good: by giving patients reason to mistrust mental health professionals, as well as caregivers, it causes them to become alienated. Coercion may further reduce patients' adherence to treatment, such as taking prescribed medication. In a New Zealand study on patients' perception of coercion on admission to acute psychiatric services, it was found that 'those admitted to hospital under the legislation feel loss of autonomy and are more likely than voluntary admissions to perceive hospitalisation as coercive. ${ }^{[11]}$ This involuntary admission has distressing psychological and physical effects on the patient. Patients are often forced to take medication and incarcerated against their will for long periods of time, which exposes them to a host of social- and life-related issues such as social isolation and stigmatisation. ${ }^{[12]}$

Many mental health professionals argue that coercion is sometimes necessary and an important part of ensuring that patients receive the proper care, particularly when patients are too ill to understand that they might need it, or might attempt to harm themselves or others. ${ }^{[13]}$ Even though it is internationally recognised that the majority of mental healthcare users should be treated voluntarily, under circumstances where the patients might cause significant distress and be a potential danger to themselves or others, it remains international practice that they might be hospitalised against their will. ${ }^{[14]}$

\section{First-person accounts of hospitalisation}

As de la Cour points out, 'Despite the extensive historical literature on asylums, few studies explore patients' perceptions of psychiatric hospitalisation, particularly what patients themselves thought about the facilities. ${ }^{[15]}$ The attitude towards hospitalisation held by those diagnosed with schizophrenia is an area of research that has been relatively ignored. The majority of studies tend to focus on staff members' perceptions of hospitalisation, leaving out the patients' perceptions completely. ${ }^{[1]}$ First-time experiences of suddenly being in an enclosed environment, with other patients who are unpredictable and behaving abnormally, could be extremely traumatic. ${ }^{[4]}$ In a 2009 study, $66 \%$ of participants $(n=31)$ said their first psychiatric admission was most distressing. ${ }^{[16]}$ To date, there is a lack of studies focusing on the traumatic effects of acute psychosis and admissions. McGorrey et al. ${ }^{[4]}$ suggest that the experience of psychiatric hospitalisation might be a direct cause of post-traumatic stress disorder (PTSD) within psychosis, following their finding that the prevalence of PTSD was $46 \%$ at 3 months of hospitalisation and $36 \%$ at 11 months after hospitalisation. ${ }^{[4]}$

Patients who have a distressing first experience of admission to a psychiatric ward are less likely to remain treatment-adherent in the future, increasing the risk of relapse. ${ }^{[17]}$ In the long term, such an experience helps to alienate them from mental health services. Examining mental healthcare users' experiences of first-time admissions to a psychiatric hospital for acute psychosis will enable healthcare professionals to understand patients' first-time experiences of the psychiatric hospital, to identify the most distressing aspects of the experiences, and to develop better intervention strategies to lessen the distressing experiences and ensure more successful first-time admissions.

\section{Methods}

This research was conducted according to an interpretive phenomenological paradigm, as the main aim was to understand participants' subjective experiences. It is important to note that this qualitative study explores the experiences through the eyes of the research participants, rather than making general inferences. Nonprobability purposive sampling was used, based on a diagnosis of schizophrenia, mental stability and previous psychiatric admissions. The diagnosis was reported by the head of the halfway facility from which participants were recruited. Such heads do not formally diagnose the patients themselves, but report the latest formal diagnosis of the patient from their current consulting psychiatrist. No other criteria such as age, gender or race were employed, as this would have made it challenging to obtain a sample. Participants were all South African citizens, white and between the ages of 24 and 54, and residents of the psychiatric halfway house. Five of the participants were male and 2 were female. All participants had more than 1 hospital admission and were treatment-resistant. Five out of the 7 participants had numerous hospital admissions, their first admission being on average 15 years ago. The other 2 participants had fewer hospital admissions, with their first admission averaging 3 years ago.

The semi-structured individual interviews consisted of open-ended questions, including general questions such as, 'Can you tell me a bit about your first experience of being in a psychiatric hospital?' and 'Did you think you required psychiatric treatment?' Some of these questions were adapted from a quantitative study ${ }^{[18]}$ that used closedended questions and that explored the patients' attitudes towards the first psychotic episode and the start of treatment.The length of each interview varied from $20 \mathrm{~min}$ to $45 \mathrm{~min}$. All the interviews were conducted in July 2010. This study did not aim for saturation as the experiences were not exhaustively explored, though this may be done in future research. Thematic content analysis was used to analyse the data.

While participants were asked primarily about their first-time admission and experiences of psychiatric hospitals, some participants still spoke about their overall hospital experience. It is possible that the first hospital admission was such a long time ago that participants may have conflated memories and their current memory of their first admission is coloured by their subsequent hospitalisations.

\section{Ethical considerations}

Ethical clearance was obtained through the University of the Witwatersrand, ref No. M10436. Written permission was obtained from the director of the halfway house to approach potential participants. Approval from every voluntary participant was gained through consent forms. Since this research dealt with a particularly sensitive issue, all participants had access to free counselling. Staff members at the halfway house agreed to monitor all residents who participated in the study and to provide containment should any residents encounter any negative effects from participating in the study. A follow-up interview with caregivers 6 months later indicated 
that none of the participants appeared to react adversely or require additional containment after the individual interviews.

\section{Results and discussion}

The main themes which emerged from the data collection were treatment, preconceived ideas of hospitalisation, incarceration, diagnosis of schizophrenia, stigmatisation, law enforcement and social interactions during initial hospitalisation.

\section{Treatment}

Treatment was addressed in terms of the sub-themes of medication, injections, occupational therapy and electroconvulsive treatment (ECT).

\section{Medication}

All the participants expressed extreme dislike of taking medication for the first time, either referring to side-effects or attributing a range of bad experiences to the medication, particularly when feeling they were compelled to ingest it. According to Participant R: 'I was so restless I couldn't sit still. I didn't know whether to lie down, whether to sit, whether to stand, to walk. I was just walking all over the place. I think it was haloperidol, I'm not sure. They put me on that drug and I couldn't have a moment's peace I was just up and up all the time, I couldn't relax.'

It seems possible that participants attributed a wide range of unpleasant experiences to the medication that may alternatively have been caused by either their illness, or predictable responses to unpleasant environmental or physical stimuli. This misattribution could be related to overall poor insight into their disorder. It also became apparent that none of the participants mentioned any positive effects resulting from the use of the medication, such as symptom reduction.

\section{Injections}

Four participants commented on their experiences of injection needles. Participants often vividly recalled their first experience of receiving forced injections. Needles were objects of profound fear for most: for some this seemed due to the physical pain associated with being injected, whereas for others it seemed to be more psychological. Participant Br described being afraid 'because I knew they wanted to bend my mind and change what I believed in and uh, I went in this room and some guy with the glasses on walked around with an injection needle and said, "Come, let me inject you."

Participant Br's initial psychiatric admission was involuntary. She seemed to describe the invasive nature of involuntary needle injection (in which the patient's body is forcibly penetrated) in far more 'delusional' terms than other treatment experiences - she experienced her 'mind' as being penetrated. It seems that the extent of psychotic distortion of thought is more severe at these times and it is possible that this is directly caused by intense feelings of fear and victimisation.

\section{Electroconvulsive treatment}

Since a few of the participants were older, it was expected that some would have had an experience of ECT, specifically because these participants were treatment resistant. However, only 1 participant mentioned undergoing this treatment. Participant PU reported: 'Um, if you went according to the rules you could have a normal life but if you do anything wrong you will be severely punished, you know. I had a lot of shock treatment and a lot of lumbar punctures.'

According to Participant PU, ECT was used as a method of punishment. Goffman ${ }^{[19]}$ (in 1961) and Foucault ${ }^{[20]}$ (in 1977) have stated that the official objective of psychiatric hospitals is to reform the mentally ill patient in the direction of some ideal standard. In other words, if patients conform to the norms they will be released, but if not they will be punished until they do conform and their behaviour is normalised. ${ }^{[19]}$ Therefore, the power structure within the institution supports Participant PU's statement as an accurate reflection of the social forces at work during his first hospitalisation. However, it is important to note that people who have schizophrenia may often have low insight, and that it is also possible that Participant PU did not understand the actual reason for the ECT, and therefore attributed it to punishment instead of treatment. Furthermore, it seems that ECT evoked a great fear in this particular participant, which may explain why he viewed it as persecutory.

Six participants who had not experienced ECT appeared to subscribe to the myth that it is a painful experience, even though in reality patients are given anaesthetics.

\section{Perceptions of hospitalisation \\ Preconceived ideas}

Perhaps due to the negative portrayal of mental illness and psychiatric hospitals in the media, ${ }^{[2]}$ it was found that participants already had a negative perception of psychiatric hospitalisation before their first admission. Participant R recalls, 'I thought they were places where you were strapped up and kept in a cell and drugged and they a did lot of what do they call it, you know when they do something to your head ... umm, shock treatment and that type of thing.'

\section{Attitudes after hospitalisation}

Four of the 7 participants reported that their first admission was extremely traumatic, in line with findings of other studies. ${ }^{[4]}$ Participant $\mathrm{Br}$ described it as 'terrible. It's like a hell hole, you know. I don't know what happened, my spirit left me, my mind left me, I was a zombie. That was one of my worst experiences in my life.'

However, one participant, Participant R, reported that his first hospital admission was a pleasant experience. 'My experience in the hospital was not what I had imagined. It was quite pleasant. Well, the fact that I had a roof over my head, because I didn't, I had been on the street for 7 years, on and off. Ja, you had 3 main meals, and you had teatime.' Participant PU also describes positive caring experiences and having his basic needs met by others.

Note that participants' statements are being made in hindsight, many years after the initial hospitalisation, and it is possible that attitudes towards the original experiences might have changed with time, particularly as the researcher was careful not to excessively probe, question or contradict such descriptions in order to avoid 'insisting' that the patient reflect on painful memories. However, it cannot be ignored that a number of patients may well find aspects of the first hospitalisation experience to be positive and rewarding, particularly as the experience may be associated with symptom improvement and a cessation of profound feelings of fear or anxiety. 


\section{for h $A R$ B

\section{Hospitalisation as social isolation}

The majority of the participants $(n=6)$ experienced their first admission to a psychiatric hospital as abandonment by their family members. Psychiatric hospitals usually require that family members limit contact during the first hospital admission, as the high expressed emotions of family members has been shown to negatively affect the patient. ${ }^{[2]}$ However, it is possible that this has long-standing consequences, as it appears that after the first hospitalisation, the patient may feel that family members offer inadequate support.

A majority of participants $(n=5)$ appear to have had some feelings of isolation during their first hospitalisation, often highlighting their loss of contact with society. Participant G expressed how difficult it was for him to be in a closed ward, where he felt alone, as he was surrounded by strangers and was not allowed free contact with his family and friends: 'You can't go out, there's nothing around you, and you feel isolated from everything ... When you actually get released or discharged or whatever, then you've got to start all over again, you've got to make new friends, usually'.

This disruption of social relationships may represent a major stressor or trauma indirectly resulting from psychiatric hospitalisation. The participants' experiences are concordant with literature that states that the most common experience among people with schizophrenia is a sense of a pervading loss of interpersonal relationships, loneliness and social isolation. ${ }^{[22]}$

\section{Social interaction and containment}

Some participants described the hospital as a social opportunity, where other patients were considered 'family'. They also described experiences of racial integration which appear to have been positive. For these participants, other patients seemed to help ease the feeling of isolation from their family members and friends. They also appeared to feel a sense of camaraderie with patients, providing them with support to help them through their hospital stay. Others felt supported by caring interactions with staff and others. As Participant PU, put it, 'Um, you meet friends and they sort of put you on the right track and help you. Others never go home, they stay there and they become like your family in the end there, you know. You know them very well. White and black and Indian and coloured all stick together. I had a very good Indian friend there, and he was there, he's there for the rest of his life'

\section{Diagnosis of schizophrenia}

For many participants, their first hospital admission was also when they were first formally diagnosed with schizophrenia. The results indicate that participants experienced serious vocational and social losses which they ascribed to this diagnosis. Participants spoke about their life prior to hospitalisation, emphasising that they were 'normal' and lived a 'normal life'.

According to Participant O, 'I didn't know what it [schizophrenia] was so I brushed it aside, I didn't accept it. I didn't know, I read the letter later and I didn't know what the letter meant, but I just brushed it aside and ignored it and decided to carry on my life as usual.' However, after being admitted to a psychiatric hospital, it was difficult for Participant $O$ to return to her normal life. She was seen as unfit for her job as a teacher. She was unable to take care of her children, who were removed from her custody.

\section{Stigma}

While the study did not ask any direct questions about stigmatisation, each participant spoke about their perceptions that they were experiencing this, not only with regard to being diagnosed with schizophrenia but also to being hospitalised. Having been stigmatised by others because they were diagnosed as schizophrenic and hospitalised, they then appeared to internalise the stigma and project it onto themselves.

Participant G explains, 'Well, I thought to myself that I thought that they are really crazy or something's wrong with them, I really didn't believe that there was anything wrong with me. I didn't want to believe that there is anything wrong with me. No one really explained to me what mental illnesses are. I think that my parents have a stigma, that I'm crazy or something.'

\section{Staff members}

Some participants reported negative interactions with staff members. Participant P recalls, 'They bath you and they shower you, it's embarrassing. Like ... I asked him please to get me something I need to urinate. They didn't come so I just weed on the floor. I didn't have an option and they gave me a little thick mattress like that to sleep on the floor. I just hear my voice and I say, "Please, please come and help me." They were laughing at me.'

Others seemed to have had an ambivalent relationship with staff members, although some indicated that it may have been because they were unhappy to be there and not necessarily because of the staff members. 'You can't blame the staff but you need that support', Participant Br points out. 'There's somebody from the outside world, you look up to him and you just need some comfort from them and [to be treated like] you're still a person, that's what you need from them and you do get it, you do get it but they tend to keep to themselves, the staff talk to the staff and the patients to the patients, you understand.'

Given South African hospitals' shortage of mental health practitioners, it is possible that the increased workload, with high levels of stress, fatigue and emotional exhaustion, affects both the quality and safety of care. ${ }^{[7]}$ The day-to-day work in a psychiatric setting encourages the use of self-protective behaviours such as emotional detachment, reductive labelling and retreating to administrative or other work. ${ }^{[8]}$

\section{Conclusion and recommendations}

The experience of persons diagnosed with schizophrenia of their first admission to a psychiatric hospital is an under-researched field, specifically within a South African context. Since all the participants expressed distress that they have minimal to no contact with their family members, it is recommended that more research be conducted to examine the specific activities and outcomes of family intervention programmes at psychiatric hospitals.

From the findings it appears that during their hospitalisation, participants have strong emotional requirements of the nursing staff. The nurse-patient relationship is clearly a complicated one, and the majority of the participants viewed nursing staff as being detached. This presents clinicians with an opportunity to enhance patients' experience during their hospitalisation. Research focusing on the nurse-patient relationship will help to explore its dynamics, which in turn will help to identify potential training initiatives to help nursing staff better manage this relationship. 
The use of injections, specifically as a coercive method, appeared to be one of the most traumatising experiences for study participants during their first admission to a psychiatric hospital. It is recommended that research be conducted on how patients are prepared for injections during hospitalisation and whether any formal procedure has been put in place for coercive treatment methods.

\section{References}

1. Rossberg JI, Friis S. Patients' and staff's perceptions of the psychiatric ward environment. Psychiatr Serv 2004;55(7):798-803. [http://dx.doi.org/10.1176/appi.ps.55.7.798]

2. Torrey EF. Surviving Schizophrenia: A Manual for Families, Consumers and Providers. 4th ed. New York: Harper Collins Publisher Inc, 2001.

3. Arieti S. Understanding and Helping the Schizophrenic: A Guide for Family and Friends Harmondsworth: Penguin, 1979.

4. McGorry PD, Chanen A, McCarthy E, et al. Posttraumatic stress disorder following recent-onset psychosis. An unrecognized postpsychotic syndrome. J Nerv Ment Dis 1991;179 (5):253-258. [http:// dx.doi.org/10.1097/00005053-199105000-00002]

5. Lund C, Flisher AJ. Norms for mental health services in South Africa. Soc Psychiatry Psychiat Epidemiol 2006;41(7):587-594. [http://dx.doi.org/10.1007/s00127-006-0057-z]

6. The World Health Organization Assessment Instrument for Mental Health Systems.WHO-AIMS Report on Mental Healthcare System in South Africa 2007. Geneva: WHO, 2007. http://www.who.int mental_health/evidence/south_africa_who_aims_report.pdf (accessed 10 July 2013).

7. Gerein N, Green A, Pearson S. The implications of shortages of health professionals for maternal health in sub-Saharan Africa. Reprod Health Matters 2006;14(27):40-50. [http://dx.doi.org/10.1016/ S0968-8080(06)27225-2

8. Hardcastle M, Kennard D, Grandison S, Fagin L, eds. Experiences of Mental Health In-patient Care Narratives from Service Users, Carers and Professionals. London: Routledge, 2007.

9. Pescosolido BA, Gardner CA, Lubell KM. How people get into mental health services: Stories of choice, coercion and 'muddling though' from 'first-timers'. Soc Sci Med 1998;46(2):275-286. [http:// dx.doi.org/10.1016/S0277-9536(97)00160-3]
10. Kaltiala-Heino R, Korkeila J, Tuohimäki C, Tuori T, Lehtinen V. Coercion and restrictions in psychiatric inpatient treatment. Eur Psychiatr 2000;15(3):213-219. [http://dx.doi.org/10.1016/ S0924-9338(00)00223-6]

11. McKenna BG, Simpson AIF, Laidlaw TM. Patient perception of coercion on admission to acute psychiatric services: The New Zealand experience. Int J L Psychiatry 1999;22(2):143-153. [http:// dx.doi.org/10.1016/S0160-2527(98)00039-9]

12. Awad AG, Voruganti LNP. Quality of life and new antipsychotics in schizophrenia Are patients better off? Int J Soc Psychiatr 1999;45(4):268-275. [http://dx.doi. org/10.1177/002076409904500405]

13. Lidz CW, Mulvey EP, Hoge SK, et al. Factual sources of psychiatric patients' perceptions of coercion in the hospital admission process. Am J Psychiatry 1998;155(9):1254-1260.

14. South African National Government. Mental Healthcare Act of South Africa No. 17 of 2002 Pretoria: Government Printer, 2002. http://www.info.gov.za/gazette/acts/2002 (accessed 22 July 2010)

15. de la Cour L. 'She thinks this is the Queen's Castle': Women patients' perceptions of an Ontario psychiatric hospital. Health Place 1997;3(2):131-141. [http://dx.doi.org/10.1016/S13538292(97)00007-5]

16. Beattie N, Shannon C, Kavanagh M, Mullholland K. Predictors of PTSD symptoms in response to psychosis and psychiatric admission. J Nerv Ment Dis 2009;197(1):56-60. [http://dx.doi. org/10.1097/NMD.0b013e31819273a8]

17. Schlebusch L, Luiz HA. Characteristics and post-hospital perceptions of patients refusing inpatient treatment in a South African general hospital psychiatric unit. Gen Hosp Psychiatry 1985;7(2):163-170. [http://dx.doi.org/10.1016/0163-8343(85)90030-1]

18. Haan LD, Peters B, Dingemans P, Wouters L, Linszen D. Attitudes of patients toward the first psychotic episode and the start of treatment. Schizophr Bull 2002;28(3):431-442.

19. Goffman E. Asylums. London: Pelican Books, 1961

20. Foucault, M. Discipline and Punish: The Birth of the Prison. New York: Vintage Books, 1977.

21. Stout PA, Villegas J, Jennings NA. Images of mental illness in the media: Identifying gaps in the research. Schizophr Bull 2004;30(3):543-561. [http://dx.doi.org/10.1093/oxfordjournals.schbul. a007099]

22. Marinelli RP, Orto AED. The Psychological and Social Impact of Disability. 4th ed. New York: Springer, 1999 